Kenji Tamura • Wataru Miwa · Tomoko Maruyama

Takao Sekiya $\cdot$ Yoshinori Murakami

\title{
Homozygous deletion on the chromosomal region 5q12.3 in human lines of small-cell lung cancers
}

\begin{abstract}
Loss of heterozygosity at the polymorphic loci on the long arm of chromosome 5 is observed in about $80 \%$ of human small-cell lung cancer (SCLC). Absence of inactivating mutations in the $A P C$ gene on 5q14 suggests the involvement of another tumor suppressor gene. We found a homozygous deletion of sequence tagged site sequence G73332 on 5q12.3 in 2 of 12 human SCLC cell lines, Lu130 and Lu134. One copy of chromosome $5 q$ was lost in these cell lines, and the remaining allele had a deletion in a more restricted region. A polymerase chain reaction-based analysis of yeast artificial chromosome, bacterial artificial chromosome (BAC), and lambda-phage clones narrowed the region of homozygous deletion to a fragment cloned within one BAC. Sequencing analysis revealed that a DNA fragment of approximately $25 \mathrm{~kb}$ was deleted interstitially, probably because of recombination through Alu repetitive sequences in Lu130 and Lu134 cells. This deletion was not detected in normal lymphocyte DNA from 98 unrelated individuals. No candidate genes, however, were detected within this region or in the adjacent $150-\mathrm{kb}$ fragment. The absence of microsatellite instability and the presence of an interstitial deletion as well as gross chromosomal aberration suggest that the genomic integrity of Lu130 and Lu134 cells might possibly be affected by Alu-mediated recombination in addition to chromosomal instability. The identical breakpoints in Lu134 and Lu135 cells as well as the same genotypes at all 33 polymorphic loci examined on various chromosomes strongly suggest that these cell lines share the
\end{abstract}

K. Tamura $\cdot$ W. Miwa $\cdot$ T. Maruyama $\cdot$ T. Sekiya $\cdot$ Y. Murakami $(\bowtie)$ Oncogene Division, National Cancer Center Research Institute,

5-1-1 Tsukiji, Chuo-ku, Tokyo 104-0045, Japan

Tel. +81-3-3547-5295; Fax +81-3-5565-9535

e-mail: ymurakam@gan2.ncc.go.jp

T. Maruyama · Y. Murakami

Tumor Suppression and Functional Genomics Project, National

Cancer Research Institute, Tokyo, Japan

Sequence data from this article have been deposited with the DDBJ/ Gen Bank Data Libraries under Accession Numbers. G73325,

G73326, G73327, G73328, G73329, G73330, G73332, and bankit 439272. same genetic materials, at least in part, during the establishment or propagation of cell lines.

Key words Small-cell lung cancer . Chromosome $5 q$. Homozygous deletion . Tumor suppressor gene . Homologous recombination · Alu-repetitive sequence

\section{Introduction}

Small-cell lung cancer (SCLC) is one of the most malignant human tumors. Inactivation of the $p 53$ and $R B$ genes, as well as loss of heterozygosity $(\mathrm{LOH})$ on the short arm of chromosome 3 , is found in almost all SCLC tumors. Furthermore, $\mathrm{LOH}$ on $5 \mathrm{q}, 11 \mathrm{p}, 16 \mathrm{q}$, and $22 \mathrm{q}$ has been frequently observed in SCLC (D'Amico et al. 1992; Hosoe et al. 1994; Kawanishi et al. 1997). In particular, LOH on 5p13-q13 was reported in $79 \%$ of SCLC cell lines by genome-wide allelotyping, suggesting that another tumor suppressor gene in this chromosomal region is involved in human SCLC (Girard et al. 2000).

Positional cloning based on a linkage study is an established method for identifying tumor suppressor genes involved in hereditary cancer. Inactivating mutations of the gene in normal lymphocyte DNA are definite criteria to prove it to be the real tumor suppressor gene (Friend et al. 1986; Groden et al. 1991; Nishisho et al. 1991). However, linkage analysis is not available in nonhereditary cancers such as SCLC. Thus, the search for tumor suppressor genes in nonhereditary cancer is usually initiated by narrowing down the chromosomal loci that commonly show LOH in many tumor samples. However, chromosomal aberrations accumulated in tumor cells are often complicated, and it is very difficult to identify a tumor suppressor gene by $\mathrm{LOH}$ analysis alone (Murakami et al. 1995; Thiagalingam et al. 1996). Alternatively, finding a fragment of homozygous deletion within a common region of $\mathrm{LOH}$ in tumor DNA provides critical information concerning the locus for the relevant gene. The tumor suppressor DPC4 and PTEN genes were successfully isolated by the analysis of a very 
limited region of homozygous deletion, which contained only a portion of the gene (Hahn et al. 1996; Li et al. 1997; Steck et al. 1997). Several candidate tumor suppressor genes were also isolated from regions of homozygous deletion in SCLC cells (Kohno et al. 1995; Xiang et al. 1996; Dammann et al. 2000; Lerman and Minna 2000; Nishioka et al. 2000).

In the present study, we identified and characterized a DNA fragment showing homozygous deletion on $5 q$ in two human SCLC cell lines.

\section{Materials and methods}

Cancer cell lines

Eleven human SCLC cell lines (Lu130, Lu134A, Lu134B, Lu135, Lu139, Lu140, SBC1, SBC2, SBC3, SBC5, and LCMA) were obtained from the Health Science Research Resources Bank (Osaka, Japan), and two human SCLC cell lines (N231 and N417) were obtained from the American Type Culture Collection (Rockville, MD, USA). Genomic DNA was extracted by the proteinase K-phenol-chloroform method (Blin and Stafford 1976).

Southern blot analysis

Five micrograms of genomic DNA was digested with restriction endonuclease EcoRI, separated by electrophoresis in $0.7 \%$ agarose gel, and transferred onto a Hybond-N+ nylon membrane (Amersham, Cleveland, USA). Hybridization with ${ }^{32} \mathrm{P}$-labeled probes was carried out as described previously (Murakami et al. 1991).

The probes for STSs, G73325, G73326, G73327, G73328, $G 73329, G 73330$, and $G 73332$ were prepared by polymerase chain reaction (PCR) amplification using normal human lymphocyte DNA as templates. A probe for the $A K T 2$ gene was obtained as described previously (Miwa et al. 1996).

Construction of a human yeast artificial chromosome (YAC) and bacterial artificial chromosome (BAC) contig

The CITB human YAC and BAC library was obtained from Research Genetics (Huntsville, AL, USA). For isolation of YAC and BAC clones, pooled DNA was screened by PCR-based analyses. Nucleotide sequence and mapping information of sequence tagged site (STS) markers on $5 \mathrm{q}$ were obtained from the National Center for Biotechnology Information and the Genome Data Base. YAC DNA was extracted as described previously (Murakami et al. 1998), whereas BAC DNA was extracted from a 100-ml culture of bacteria using a Qiagen Plasmid Midi Kit (Qiagen, Tokyo, Japan).

Radiation hybrid mapping of the fragment $G 73332$

A chromosomal locus for the fragment G73332 was assigned by PCR-based analysis of DNA from the
White-head Institute radiation hybrid panel and the Stanford G3 radiation hybrid panel established by the Stanford Human Genome Center (Stewart et al. 1997; http://www-shgc.stanford.edu/).

Isolation of lambda phage clones

The lambda phage library of human genomic DNA was obtained from the Clontech Corporation (Palo Alto, CA, USA). One million phage clones were screened by hybridization with a ${ }^{32} \mathrm{P}$-labeled 673332 fragment. The DNA of phage clones was partially digested with Hind III, separated with agarose gel electrophoresis, and used for construction of the restriction map. A nucleotide sequence of the end portion of the lambda phage clones was determined by a Bigdye DNA cycle sequencing kit (PE Applied Biosystems, CA, USA).

\section{Long-range PCR}

DNA fragments flanked by STS markers were amplified by PCR with LA-Taq DNA polymerase (Takara, Kyoto, Japan) using the DNA from a BAC clone, 455i12, as a template. PCR was carried out with an initial denaturing step $\left(94^{\circ} \mathrm{C}\right.$ for $\left.3 \mathrm{~min}\right)$, followed by a 40 -cycle reaction $\left(94^{\circ} \mathrm{C}\right.$ for $1 \mathrm{~min}, 68^{\circ} \mathrm{C}$ for $15 \mathrm{~min}$ ).

Nucleotide sequencing of a $25-\mathrm{kb}$ fragment

Five DNA fragments covering the $25-\mathrm{kb}$ fragments were amplified by PCR and cloned into a plasmid vector, pGEM2. The nucleotide sequences of each cloned DNA were determined by bidirectional walking from the vector sequences of the plasmid.

\section{Results}

Homozygous deletion in SCLC cell lines

LOH on $5 q$ was often observed in human SCLC (D'Amico et al. 1992; Hosoe et al. 1994). To narrow down the region harboring candidate tumor suppressor genes, 18 STS markers mapped on 5q were examined for homozygous deletion by PCR analysis in 12 human SCLC cell lines. These STSs included WI-3663, IB2013, D5S2072, AFMA296ZE5, D5S2089, D5S603, D5S647, G16960, G16952, D5S2121, AFMC020ZD1, D5S1967, GATA-P16209, WI-7856, D5S629, WI-6225, WI-6159, and G73332. One of the STS sequences, G73332, was not amplified in two cell lines, Lu130 and Lu134 (data not shown). In addition, these two cell lines showed hemizygosity at all other polymorphic markers on $5 \mathrm{q}$, indicating that one copy of chromosome $5 \mathrm{q}$ was simply lost in these cell lines. The STS, G73332, was originally established as a DNA fragment flanked by a Not I restriction site through restriction landmark genome scanning analysis and roughly mapped on $5 q$ by radiation hybrid 


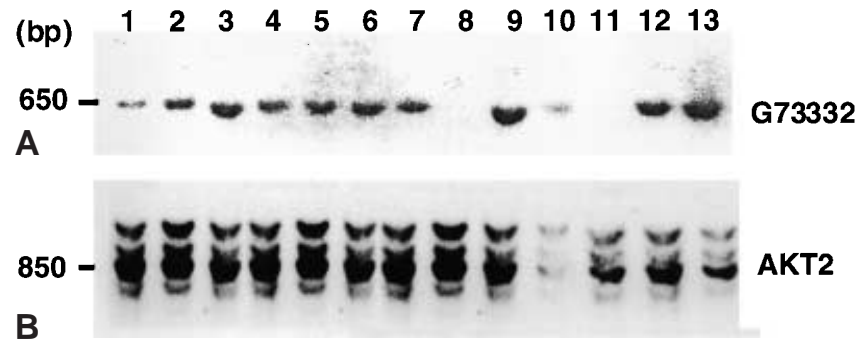

Fig. 1A,B. Homozygous deletion of the Sequence tagged site (STS) sequence, G73332, on chromosome 5q12 in human Small-cell lung cancer (SCLC) cell lines. Southern blot analysis of the G73332 (A) and the AKT2 on 19q13 (B). Lane 1, SBC1; lane 2, SBC2; lane 3, SBC3; lane 4, SBC5; lane 5, LCMA; lane 6, N231; lane 7, N417; lane 8, Lu130; lane 9, Lu135; lane 10, Lu139; lane 11, Lu134; lane 12, Lu140; lane 13, normal lung

clones (Miwa et al. 1996). Absence of the $G 73332$ sequence in the DNA from Lu130 and Lu134 cells was confirmed by Southern blot analysis using a 780-bp fragment containing G73332 as a probe (Fig. 1).

Mapping of the G73332 fragment on 5p12

For detailed mapping of chromosomal loci for the $G 73332$ fragment, the YAC library was screened by PCR for the presence of the $G 73332$ sequence. As summarized in Fig. $2 \mathrm{~A}$, four YAC clones, 922g9, 811h12, 785a9, and 771 $\mathrm{g} 2$, gave positive signals derived from $G 73332$. Because a DNA fragment flanked by two STS markers, D5S2089 and D5S2121, was commonly retained in these four YAC clones, $G 73332$ appeared to be located within this region of approximately $2 \mathrm{cM}$.

Analyses of radiation hybrids from the Whitehead Institute also mapped the $G 73332$ to the region flanked by D5S2089 and D5S2121, where a marker, D5S647, showed the highest linkage to the G73332 fragment (Fig. 2B). Another panel of radiation hybrids from Stanford University mapped the G73332 around three STS markers, D5S603, G16960, and G16952 (Fig. 2C). All these STS sequences were amplified by PCR in the DNA from Lu130 and Lu134 cells, indicating that a region of homozygous deletion was restricted to a relatively small fragment just around the STS, G73332.

Interstitial deletion around the $G 73332$ fragment

Screening of the lambda phage library with a radiolabeled G73332 fragment as a probe revealed that three phage clones, KL3, KL4, and KL5, contained the G73332 sequence. A restriction map of these clones by Hind III showed that most of the inserts overlapped one another (Fig. 3A). The end sequences of KL4 and KL5 were then determined, and four STSs ( $G 73325, G 73326, G 73329$, and G73330) were subsequently established. Southern blot analysis revealed that three STSs, G73326, G73329, and G73330, were absent, whereas one STS, G73325, was

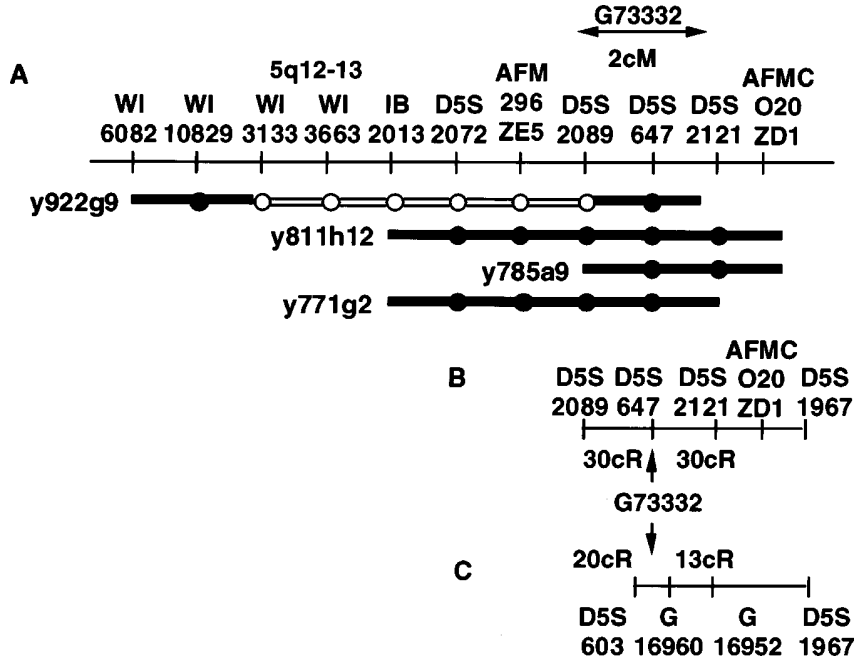

Fig. 2A-C. Mapping of the G73332 on chromosome 5q12. A Alignment of four yeast artificial chromosome (YAC) clones carrying the STS sequence, G73332. The order of the STS markers was based on the information from GeneBank. Closed and open circles indicate the presence and the absence of the STS sequences on the YAC determined by polymerase chain reaction-based analysis, respectively. B, C Mapping results of $G 73332$ on the radiation hybrid panels from the Whitehead Institute $\mathbf{B}$ and Stanford University, G3 C

present in the DNA from both Lu130 and Lu134 cells (Fig. 3B). These results indicate that one of the break points resides within the 3.9-kb fragment between two STSs, G73325 and G73330, both in Lu130 and Lu134 cells (Fig. $3 \mathrm{~A})$.

To identify the other breakpoint, we screened a BAC library for the presence of $G 73332$ as well as its flanking STS sequences, and seven BAC clones were subsequently obtained, containing the whole inserts of both lambda phage clones, L4 and L5 (Fig. 4A). The end sequences of one of the BAC clones, 455i12, were determined, and a pair of new STS markers, G73327 and G73328, was established. PCRbased analysis revealed that both $G 73327$ and $G 73328$ are present in Lu130 and Lu134 cells, indicating that a fragment showing homozygous deletion is restricted within a BAC clone, 4555i12. The order of the relevant STS markers was then determined by long-range PCR, which was designed to encompass the pairs of STS sequences. As shown in Fig. 4B, DNA fragments of $8.7 \mathrm{~kb}$ and $7.3 \mathrm{~kb}$ were amplified by a pair of primers from STSs, $G 73327$ and $G 73329$, and those from $G 73326$ and $G 73327$, respectively. The relative order of these STS markers was subsequently determined, as shown in Fig. 4A. These analyses mapped the second breakpoint to a fragment of $7.3 \mathrm{~kb}$ flanked by $G 73326$ and G73327 and defined the region of homozygous deletion to a fragment of approximately $25 \mathrm{~kb}$ flanked by $G 73325$ and G73327.

Next, the DNA sequence of $25 \mathrm{~kb}$ covering the region of homozygous deletion was determined by using DNA from a BAC clone, 455i12, as a template. PCR-based analyses defined the breakpoints into the Alu-repetitive sequence located between $G 73325$ and $G 73330$ and that between $G 73326$ and $G 73327$, respectively. PCR by a pair of primers 
G73332

KL3

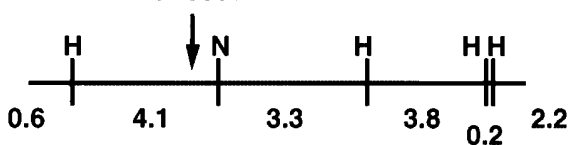

$14.2 \mathrm{~kb}$

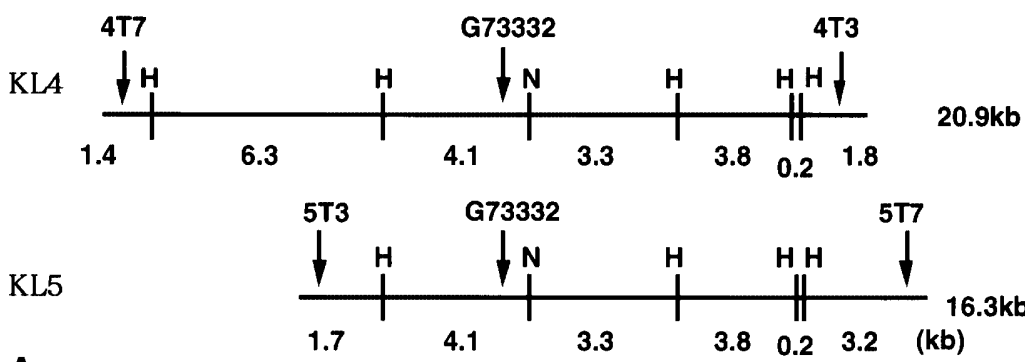

1233456

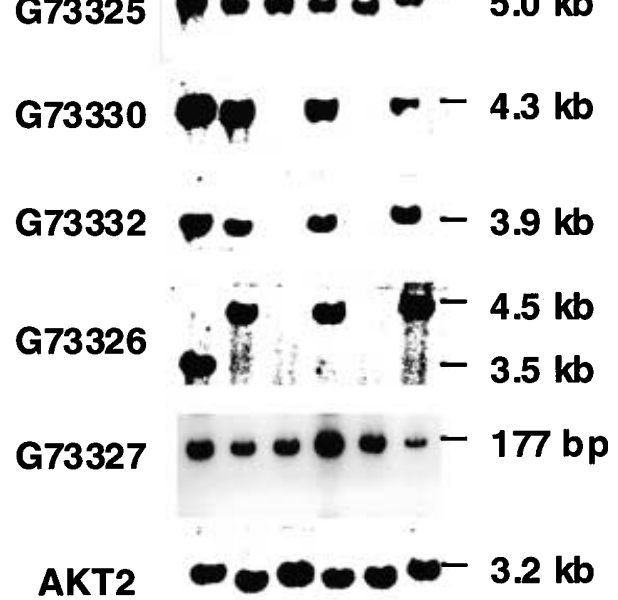

Fig. 3A,B. Structural analysis of the DNA fragments adjacent to the G73332. A Restriction map of the three lambda-phage clones carrying the STS sequence, $G 73332$. $H$ and $N$ indicate the restriction sites of Hind III and Not I, respectively. The size of the inserts as well as the distance between the restriction sites was determined on the basis of
Southern blot analysis. The end sequences of the lambda-phage clones, KL4 and KL5, were determined, and pairs of STS sequences, G73325 and $G 73329$ and $G 73330$ and $G 73326$, were established. B Southern blot analysis and PCR-based analysis (G73327) of the five STS markers adjacent to the G73332 in Lu130 and Lu134 cells

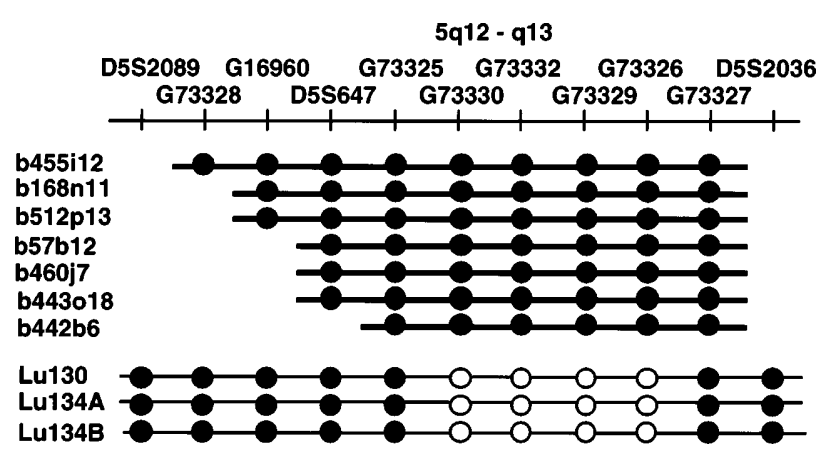

A
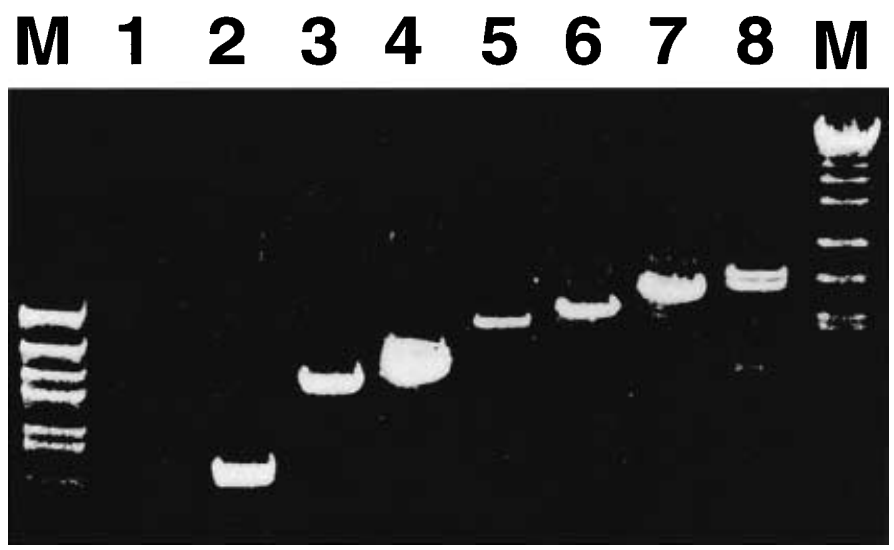

B

presence and the absence of the STS sequences, respectively. B Longrange PCR analysis between the STS markers. The pairs of primers and the size of the PCR products were as follows: Lane 1, no DNA; lane 2, G73325 and $G 73330,3.9 \mathrm{~kb}$; lane 3, G73330 and G73332, $6.3 \mathrm{~kb}$; lane 4, $G 73327$ and $G 73326,7.3 \mathrm{~kb}$; lane 5, G73332 and G73329, 8.4 kb; lane 6, G73327 and G73329, $8.7 \mathrm{~kb}$; lane 7, G73332 and G73326, $9.8 \mathrm{~kb}$; lane 8, $G 73325$ and $G 73332,10.2 \mathrm{~kb}$. $M$, Size marker

This interstitial deletion was not observed in normal lymphocyte DNA from 98 unrelated individuals, suggesting that this was more likely to be a mutation accumulated in these cell lines rather than a polymorphic change (data not shown). Lu130 and Lu134 cells harbored not only identical regions of homozygous deletion, but also the same genotypes in 17 polymorphic loci examined on chromosome 5 . Furthermore, genotyping analysis of Lu130 and Lu134 at a total of 16 different chromosomal loci showed the same results, indicating that these two are the same cell lines, although originally established from different SCLC tumors from two distinct patients (data not shown). 


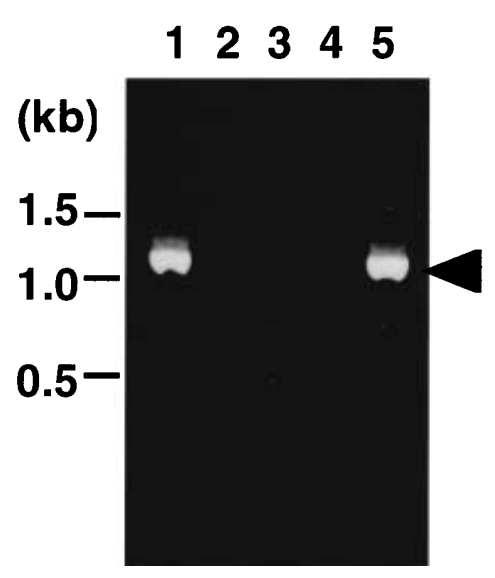

A

\begin{tabular}{|c|c|}
\hline 5 'BP Alu & $5^{\prime}$-TGTTTTAAATTTAAAAAAATTTTCGTCTGGGCATGGTGGCTCACGCCTGTAATCCCAG \\
\hline & $* * * * * * * * * * * * * * * * * * * *$ \\
\hline 3'BP Alu & $5^{\prime}$-GTGGTTCACGCCTGCAATCCCTG \\
\hline 5'BP Alu & CACTTTGGGAGGCCGAGGCAGGTGGATCAAAAGGTCAAGAGATTGAGACCATCCTGGCCA \\
\hline & 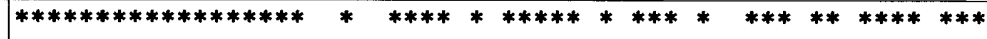 \\
\hline 3'BP Alu & CACTTTGGGAGGCCGAGGTGGCCGGATCACAAGGTTAGGAGTTCCAGATCAGCCTGACCA \\
\hline 5'BP Alu & ACATGGTGAAACCCCGTCTCTACTAAAAATACAAAAATTAGCTGGGTATGGTGGCGGGTG \\
\hline & ***** $\quad * * * * * * * * * * * * * * * * * * * * * * \quad * * \quad * * * * * * * * * * * * * \quad * * \quad * * * * * *$ \\
\hline 3'BP Alu & ACATGAAGAAACCCCGTCTCTACTAAAAACACGAAAATTAGCTGGGCATAGTGGCGCACA \\
\hline 5'BP Alu & CCGATAGTCCCAGCTACTTGGAAGGCTGAGGCAGGAGAATCACTTGAAAC-CGGAGGTGG \\
\hline & ** $\quad * * * * * * * \quad * * * * * * * \quad * * * * * * * * \quad * * * * * * * * * * * *) \quad * * * * * \quad *$ \\
\hline 3'BP Alu & CC---TGT--AAGCTACTCGGGAGGCCGAGGCAGGCGAATCACTTGAACCTGGGAGGCAG \\
\hline 5'BP Alu & ATTTTGCAGTGAGCAAAGATCACACCACTGCACTCCAACCTGGGAGACAGAGTGAGACTC \\
\hline & 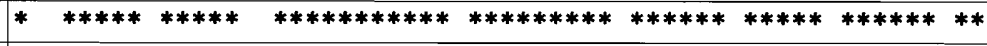 \\
\hline 3'BP Alu & AGGTTGCACTGAGCTGAGATCACACCATTGCACTCCAGCCTGGGTGACAGGGTGAGAGTC \\
\hline 5'BP Alu & TGTCTCAAAAACAAACAGACAAAAAAATTTCTGATTTCCAGGAGCCACACATTTGAC-3' \\
\hline & $* * * * * * * * * * * * * * * * * * * * * * *$ \\
\hline 3'BP Alu & 'TGTCTCAAAAAAAAAAAAAAAAGAAAAGAAAAAAGAAAGAAA-3' \\
\hline
\end{tabular}

B

Fig. 5A,B. Identification of the breakpoint of the interstitial deletion. A The results of the long-range PCR using a pair of primers flanked by the breakpoint. Lane 1, Lu130; lane 2, Lu135; lane 3, Lu139; lane 4, Lu140; lane 5, Lu134. B Alignment of the Alu-Sp subfamily sequence surrounding the $5^{\prime}$ breakpoint $\left(5^{\prime} B P\right)$ and the Alu-Sc subfamily

Search for genes in the region of homozygous deletion

To find a gene within the deleted region, we screened cDNA libraries derived from adult lung, fetal lung, and adult brain tissues by using several genomic DNA segments as probes that exclude repetitive sequences from the relevant region. No cDNA clones, however, were identified by screening of approximately one million clones.

Next, DNA sequences that we had determined (bankit 439272) as well as those obtained from the Genome Database (NT 006796) were examined for the presence of a gene or gene by using several public databases and gene prediction programs including Grail, Hexon, and Genescan. However, no sequences were matched with any known genes, spliced expressed sequence tags (ESTs), or predicted gene sequences. Analysis of a DNA sequence of $250 \mathrm{~kb}$ around the region of the homozygous deletion from NT 006796 revealed that the LY64 and KIAA0303 genes were located at the region of about $150 \mathrm{~kb}$ distal to the STS, G73332. However, the expression pattern of the two genes was not changed in Lu130 and Lu134 cells in comparison with normal lung or brain tissues (data not shown). sequence surrounding the $3^{\prime}$ BP. Sequences in the vicinity of the breakpoint are underlined, and those at the junction are doubleunderlined. Asterisks indicate identical nucleotides between the two Alu sequences

\section{Discussion}

$\mathrm{LOH}$ on $5 \mathrm{p} 13-\mathrm{q} 13$ is one of the most frequent occurrences in human SCLC. We demonstrated the homozygous deletion of a DNA fragment within this region, $5 \mathrm{q} 12.3$, in 2 of 12 SCLC cell lines, Lu130 and Lu134. One copy of chromosome $5 \mathrm{q}$ was lost, whereas the remaining allele showed an interstitial deletion of $25 \mathrm{~kb}$, probably by recombination through Alu repetitive sequences in both cell lines. The breakpoint was defined within identical sequences of $12 \mathrm{bp}$ located at the two Alu repetitive elements. The absence of the deletion in 196 normal alleles suggests that this deletion may be a mutation specific to these cancer cells rather than a polymorphic change. $G 73332$ is mapped adjacent to the NotI recognition site, which is often associated with the promoter regions of genes. However, we failed to identify any candidate gene fragments, including ESTs, within the deleted region or in the vicinity of about $250 \mathrm{~kb}$. An unidentified gene around this region might possibly be affected by the homozygous deletion. In fact, several candidate tumor suppressor genes have been identified from the regions of homozygous deletion caused by recombination through the Alu sequence in SCLC cells (Kohno et al. 1995; Xiang et al. 1996; Dammann et al. 2000; Nishioka et al. 2000). Alu-mediated deletions of specific gene loci of $0.8-24 \mathrm{~kb}$ have also been reported as germline mutations 
in several tumor suppressor genes, including $W T 1, R B 1$, $B R C A 1$, and APC (Tadokoro et al. 1992; Rothberg et al. 1997; Swensen et al. 1997; Su et al. 2000). Alternatively, this deletion might not be a causative mutation for carcinogenesis or progression but a random genetic alteration accumulated in cancer cells. In particular, it could occur when two cell lines, Lu130 and Lu134, are not independent but, rather, identical cell lines. In the series of analyses in the present study, microsatellite instability was not detected in Lu130 and Lu134 cells. In contrast, gross chromosomal abnormalities, including hemizygosity in several chromosomes, were observed in these cell lines, suggesting that chromosomal instability is one of the driving forces toward malignant progression. In addition, recombination through Alu repetitive sequences might possibly be involved in the disruption of the genomic integrity in these SCLC cell lines.

Lu130 cells and two sublines of Lu134 cells, Lu134A and Lu134B, showed identical breakpoints on 5q12.3 and also carried the same genotypes at all 33 polymorphic loci analyzed on various chromosomes. We examined another batch of Lu130, Lu134A, and Lu134B cells from a supplier and obtained the same results. Futami et al. (1995) also reported a relatively rare but identical mutation of the RET gene on chromosome 10q in Lu130 and Lu134 cells. These results indicate that Lu130 and lu134 cells share the same genetic materials, at least in part, during the establishment or propagation of cell lines.

Acknowledgments We thank Jun Yasuda for his helpful participation in discussions and Kana Isogai for her technical assistance. This work was supported in part by a Grant-in-Aid for the Second Comprehensive 10-Year Strategy for Cancer Control from the Japanese Ministry of Health, Labor, and Welfare and by a Grant-in-Aid for Special Projects for Cancer Research from the Japanese Ministry of Education, Culture, Science, Sports, and Technology. K.T. and W.M. are recipients of Research Resident Fellowships from the Japanese Foundation for the Promotion of Cancer Research.

\section{References}

Blin N, Stafford DW (1976) A general method for isolation of high molecular weight DNA from eukaryotes. Nucleic Acids Res 3:23032308

D’Amico D, Carbone DP, Johnson BE, Meltzer SJ, Minna JD (1992) Polymorphic sites within the $M C C$ and $A P C$ loci reveal very frequent loss of heterozygosity in human small cell lung cancer. Cancer Res 52:1996-1999

Dammann R, Li C, Yoon JH, Chin PL, Bates S, Pfeifer GP (2000) Epigenetic inactivation of a RAS association domain family protein from the lung tumor suppressor locus 3p21.3. Nat Genet 25:315319

Friend SH, Bernards R, Rogelj S, Weinberg RA, Rapaport JM, Albert DM, Dryja TP (1986) A human DNA segment with properties of the gene that predisposes to retinoblastoma and osteosarcoma. Nature 323:643-646

Futami H, Egawa S, Tsukada T, Maruyama K, Bandoh S, Noguchi M, Yamaguchi K (1995) A novel somatic point mutation of the RET proto-oncogene in tumor tissues of small cell lung cancer patients. Jpn J Cancer Res 86:1127-1130

Girard L, Zochbauer-Muller S, Virmani AK, Gazdar AF, Minna JD (2000) Genome-wide allelotyping of lung cancer identifies new regions of allelic loss, differences between small cell lung cancer and non-small cell lung cancer, and loci clustering. Cancer Res 60:4894 4906
Groden J, Thliveris A, Samowitz W, Carlson M, Gelbert L, Albertsen H, Joslyn G, Stevens J, Spirio L, Robertson M, White RL (1991) Identification and characterization of the familial adenomatous polyposis coli gene. Cell 66:589-600

Hahn SA, Schutte M, Hoque AT, Moskaluk CA, da Costa LT, Rozenblum E, Weinstein CL, Fischer A, Yeo CJ, Hruban RH, Kern SE (1996) DPC4, a candidate tumor suppressor gene at human chromosome 18q21.1. Science 271:350-353

Hosoe S, Ueno K, Shigedo Y, Tachibana I, Osaki T, Kumagai T, Tanio Y, Kawase I, Nakamura Y, Kishimoto T (1994) A frequent deletion of chromosome 5q21 in advanced small cell and non-small cell carcinoma of the lung. Cancer Res 54:1787-1790

Kawanishi M, Kohno T, Otsuka T, Adachi J, Sone S, Noguchi M, Hirohashi S, Yokota J (1997) Allelotype and replication error phenotype of small cell lung carcinoma. Carcinogenesis 18:20572062

Kohno T, Otsuka T, Takano H, Yamamoto T, Hamaguchi M, Terada M, Yokota J (1995) Identification of a novel phospholipase C family gene at chromosome $2 \mathrm{q} 33$ that is homozygously deleted in human small cell lung carcinoma. Hum Mol Genet 4:667-674

Lerman MI, Minna JD (2000) The 630-kb lung cancer homozygous deletion region on human chromosome 3 p21.3: identification and evaluation of the resident candidate tumor suppressor genes. The International Lung Cancer Chromosome 3p21.3 Tumor Suppressor Gene Consortium. Cancer Res 60:6116-6133

Li J, Yen C, Liaw D, Podsypanina K, Bose S, Wang SI, Puc J, Miliaresis C, Rodgers L, McCombie R, Bigner SH, Giovanella BC, Ittmann M, Tycko B, Hibshoosh H, Wigler MH, Parsons R (1997) PTEN, a putative protein tyrosine phosphatase gene mutated in human brain, breast, and prostate cancer. Science 275:1943-1947

Miwa W, Yasuda J, Murakami Y, Yashima K, Sugano K, Sekine T, Kono A, Egawa S, Yamaguchi K, Hayashizaki Y, Sekiya T (1996) Isolation of DNA sequences amplified at chromosome 19q13.1q13.2 including the $A K T 2$ locus in human pancreatic cancer. Biochem Biophys Res Commun 225:968-974

Murakami Y, Hayashi K, Sekiya T (1991) Detection of aberrations of the $p 53$ alleles and the gene transcript in human tumor cell lines by single-strand conformation polymorphism analysis. Cancer Res 51:3356-3361

Murakami YS, Brothman AR, Leach RJ, White RL (1995) Suppression of a malignant phenotype in a human prostate cancer cell line by fragments of normal chromosomal region 17q. Cancer Res 55:3389-3394

Murakami Y, Nobukuni T, Tamura K, Maruyama T, Sekiya T, Arai Y, Gomyou H, Tanigami A, Ohki M, Cabin D, Frischmeyer P, Hunt P, Reeves RH (1998) Localization of tumor suppressor activity important in non-small cell lung carcinoma on chromosome 11q. Proc Natl Acad Sci USA 95:8153-8158

Nishioka M, Kohno T, Takahashi M, Niki T, Yamada T, Sone S, Yokota J (2000) Identification of a 428-kb homozygously deleted region disrupting the $S E Z 6 L$ gene at 22q12.1 in a lung cancer cell line. Oncogene 19:6251-6260

Nishisho I, Nakamura Y, Miyoshi Y, Miki Y, Ando H, Horii A, Koyama K, Utsunomiya J, Baba S, Hedge P (1991) Mutations of chromosome 5q21 genes in FAP and colorectal cancer patients. Science 253:665-669

Rothberg PG, Ponnuru S, Baker D, Bradley F, Freeman AI, Cibis GW, Harris DJ, Heruth DP (1997) A deletion polymorphism due to AluAlu recombination in intron 2 of the retinoblastoma gene: association with human gliomas. Mol Carcinog 19:69-73

Steck PA, Pershouse MA, Jasser SA, Yung WK, Lin H, Ligon AH, Langford LA, Baumgard ML, Hattier T, Davis T, Frye C, Hu R, Swedlund B, Teng DH, Tavtigian SV (1997) Identification of a candidate tumor suppressor gene, $M M A C 1$, at chromosome 10q23.3 that is mutated in multiple advanced cancers. Nat Genet 15:356362

Stewart EA, McKusick KB, Aggarwal A, Bajorek E, Brady S, Chu A, Fang N, Hadley D, Harris M, Hussain S, Lee R, Maratukulam A, O'Connor K, Perkins S, Piercy M, Qin F, Reif T, Sanders C, She X, Sun W-L, Tabar P, Voyticky S, Cowles S, Fan J-B, Mader C, Quackenbush J, Myers RM, Cox DR (1997) An STS-based radiation hybrid map of the human genome. Genome Res 7:422-433

Su LK, Steinbach G, Sawyer JC, Hindi M, Ward PA, Lynch PM (2000) Genomic rearrangements of the $A P C$ tumor-suppressor gene in familial adenomatous polyposis. Hum Genet 106:101-107 
Swensen J, Hoffman M, Skolnick MH, Neuhausen SL (1997) Identification of a 14-kb deletion involving the promoter region of BRCA1 in a breast cancer family. Hum Mol Genet 6:1513-1517

Tadokoro K, Fujii H, Ohshima A, Kakizawa Y, Shimizu K, Sakai A, Sumiyoshi K, Inoue T, Hayashi Y, Yamada M (1992) Intragenic homozygous deletion of the WT1 gene in Wilms' tumor. Oncogene 7:1215-1221

Thiagalingam S, Lengauer C, Leach FS, Schutte M, Hahn SA, Overhauser J, Willson JK, Markowitz S, Hamilton SR, Kern SE,
Kinzler KW, Vogelstein B (1996) Evaluation of candidate tumor suppressor genes on chromosome 18 in colorectal cancers. Nat Genet 13:343-346

Xiang RH, Hensel CH, Garcia DK, Carlson HC, Kok K, Daly MC, Kerbacher K, van den Berg A, Veldhuis P, Buys CH, Naylor SL (1996) Isolation of the human semaphorin III/F gene $(S E M A 3 F)$ at chromosome 3p21, a region deleted in lung cancer. Genomics 32:3948 